\title{
Uterine Corpus Leiomyosarcoma by AJCC v8 Stage
}

National Cancer Institute

\section{Source}

National Cancer Institute. Uterine Corpus Leiomyosarcoma by A/CC v8 Stage. NCI

Thesaurus. Code C139870.

A term that refers to the staging of uterine corpus leiomyosarcoma according to the American Joint Committee on Cancer, 8th edition. 